\title{
Distribución espacio-temporal de hormigas en un gradiente de luz, dentro de un sistema agroforestal de café, en Turrialba, Costa Rica
}

\author{
Edgar H. Varón ${ }^{1}$, Paul Hanson ${ }^{2}$, John T. Longino ${ }^{3}$, Olger Borbón ${ }^{4}$, Manuel Carballo ${ }^{5}$ \\ \& Luko Hilje ${ }^{5}$ \\ 1 Investigador Ph.D. Asistente. CORPOICA C.I. Nataima. Chicoral (Tolima). Colombia. Km 9 Vía Espinal, Ibagué. \\ evaron@corpoica.org.co; evaron@catie.ac.cr. Fax: (506) 558-2043. \\ 2 Escuela de Biología, Universidad de Costa Rica, San José, Costa Rica. \\ 3 The Evergreen State College, Olympia, Washington, EEUU. \\ 4 Centro de Investigaciones en Café (CICAFE), Heredia, Costa Rica. \\ 5 Departamento de Agricultura y Agroforestería, CATIE, Turrialba, Costa Rica.
}

Recibido 16-V-2003. Corregido 05-VIII-2006. Aceptado 11-IV-2007.

\begin{abstract}
Temporal and spatial distribution of ants in a light gradient, in a coffee agroforestry system, Turrialba, Costa Rica. Shade trees are frequently present in coffee (Coffea arabica L.) agroforestry systems of Mesoamerica. These systems can harbor a rich entomofauna, including ants, which could be predators of key pests in these systems. However, the role of shade on the distribution and abundance of these ants is unknown, yet such knowledge could suggest guidelines for manipulating certain environmental conditions of their habitat, thereby achieving their conservation and increase. Therefore, we studied the effect of shade on the spatial and temporal distribution of three ant species (Solenopsis geminata, Pheidole radoszkowskii and Crematogaster curvispinosa) that may prey on the coffee berry borer, Hypothenemus hampei (Coleoptera: Scolytidae), and the mahogany shootborer, Hypsipyla grandella (Lepidoptera: Pyralidae). To do this, abundance was evaluated across a sun-shade gradient in a coffee plantation with four alternate plots (from pure sun to total shade) in Turrialba, Costa Rica. In the community that was studied 28 species of ants were collected, of which S. geminata was the dominant species (79\% of the total individuals), followed by P. radoszkowskii (16\%). S. geminata and C. curvispinosa preferred sunny areas, while P. radoszkowskii showed no defined preference. Likewise, with respect to location, $S$. geminata predominated in the soil, while P. radoszkowskii and C. curvispinosa predominated in coffee bushes. Rev. Biol. Trop. 55 (3-4): 943-956. Epub 2007 December, 28.
\end{abstract}

Key words: Coffee, shade, Solenopsis geminata, Pheidole radoszkowskii, Crematogaster curvispinosa, Costa Rica, agroforestry systems.

En Mesoamérica es común la presencia de árboles de sombra en los sistemas agroforestales de café (Coffea arabica L.), los cuales cumplen varias funciones agronómicas sensu stricto, tales como la creación de un microclima favorable para el cultivo, la incorporación de materia orgánica de la hojarasca, la fijación de nitrógeno, la circulación de nutrimentos y la disminución de la erosión del suelo (Beer et al. 1998). Asimismo, pueden albergar una biodiversidad animal valiosa, en términos económicos y/o ecológicos, incluyendo a ciertos grupos de insectos de los órdenes Coleoptera (Nestel et al. 1992), Homoptera (Rojas et al. 2001a, b) e Hymenoptera (Perfecto y Snelling 1995, Perfecto et al. 1997).

En Costa Rica, entre los representantes del orden Hymenoptera comunes en cafetales destacan las hormigas, y actualmente se dispone de buenos inventarios sobre comunidades de hormigas en cafetales del Valle Central y de la vertiente del Caribe (Perfecto y Snelling 1995, Barbera et al. 2004). A pesar de sus hábitos alimenticios generalistas (Hölldobler y Wilson 
1990, Longino y Hanson 1995), algunas especies pueden depredar a insectos herbívoros, por lo que podrían ser útiles en programas de manejo integrado de plagas del café, como la broca del café, Hypothenemus hampei (Ferrari) (Varón et al. 2004) y el barrenador de las meliáceas, Hypsipyla grandella (Zeller) (Varón et al. 2005) que ataca a los cedros y caobas sembrados a veces en cafetales.

En contraste con el control biológico inundativo, el cual implica altos costos y dificultades logísticas para la crianza masiva de enemigos naturales de las plagas, con las hormigas depredadoras se podría recurrir a la manipulación de ciertas condiciones de su hábitat para lograr su conservación e incremento. Esto se ha hecho exitosamente con otras especies de hormigas en otros sistemas como cultivos de naranja (Huang y Yang 1987), camote (Perfecto y Castiñeiras 1998) y otros más (Way y Khoo 1992). Sin embargo, para mejorar dichas condiciones es un requisito estudiar las interacciones de las especies promisorias de hormigas dentro de los agroecosistemas cafetaleros, tales como sus respuestas a factores ambientales y bióticos críticos, incluyendo sus sitios de alimentación y anidación.

El objetivo de esta investigación fue determinar el efecto de los árboles de sombra sobre la distribución espacial y temporal de tres especies de hormigas (Solenopsis geminata, Pheidole radoszkowskii y Crematogaster curvispinosa) como potenciales depredadoras de H. hampei o H. grandella.

\section{MATERIALES Y MÉTODOS}

La investigación se realizó entre febrero y agosto de 2002, en una parcela de café (lote El Cañal), ubicada dentro de una finca comercial de la empresa Lindo, en Santa Rosa de Turrialba, $9^{\circ} 52^{\prime} \mathrm{N}$ y $83^{\circ} 38^{\prime} \mathrm{W}$, Costa Rica, en la vertiente del Caribe, a $590 \mathrm{msnm}$. Los valores anuales promedio de precipitación, temperatura y humedad relativa son de 2 $479 \mathrm{~mm}, 21.7{ }^{\circ} \mathrm{C}$ y $87 \%$, respectivamente. Aunque no existe estacionalidad marcada en la precipitación, ésta en general disminuye entre enero y abril y se incrementa en mayo, junio, julio, noviembre y diciembre.

En cuanto a las condiciones climáticas que prevalecieron durante el período de estudio, la precipitación fue considerada como equivalente para los microhábitats de sombra y sol (Fig. 1A). Por su parte, la variación térmica promedio fue baja, de apenas $1{ }^{\circ} \mathrm{C}$ al sol y $1.3{ }^{\circ} \mathrm{C}$ a la sombra, y la temperatura promedio difirió levemente entre ambos microhábitats (sombra, $21{ }^{\circ} \mathrm{C}$ y sol, 21. $5{ }^{\circ} \mathrm{C}$ ) (Fig. 1B). En cambio, la humedad relativa promedio fue levemente mayor en la sombra (82\%) que en el sol (76 \%) (Fig. 1C).

La parcela medía aproximadamente 9200 $\mathrm{m}^{2}$ y estaba dividida en dos parcelas de variedades de café separadas por un camino, una de $4580 \mathrm{~m}^{2}$ (variedad Caturra) y la otra de 4 $295 \mathrm{~m}^{2}$ (variedad Catimor 5 175); aquí se les denomina parcela I y II, respectivamente. Cada una estaba subdividida en cuatro subparcelas de aproximadamente $1100 \mathrm{~m}^{2}$, dos a pleno sol y dos con sombra de poró (Erythrina poeppigiana (Walpers) O.F. Cook, Fabaceae: Papilionoideae), dispuestas de manera alterna. Los árboles de poró fueron establecidos en 1993, en una densidad de 20 por parcela, y durante el estudio medían unos $20 \mathrm{~m}$ de altura. En ambas parcelas, las actividades agronómicas realizadas en el café durante el período de estudio incluyeron dos fertilizaciones, una poda, una deshija y dos aplicaciones de herbicida, mientras que al poró no se le realizó ninguna labor.

En cada una de las parcelas, aprovechando la alternancia de subparcelas con y sin sombra, se establecieron seis transectos de $12 \mathrm{~m}$ de longitud, aproximadamente, los cuales incluyeron varios gradientes, desde sombra total hasta pleno sol. En cada transecto hubo cuatro estaciones de muestreo, según la siguiente secuencia: a pleno sol (denominado SOL), sol parcial (SOP), sombra parcial (SMP) y sombra total (SOM) (Fig. 2).

Las tres especies de hormigas se muestrearon mediante trampas (cebo atrayente), mensualmente. Estas consistieron en cuadritos 

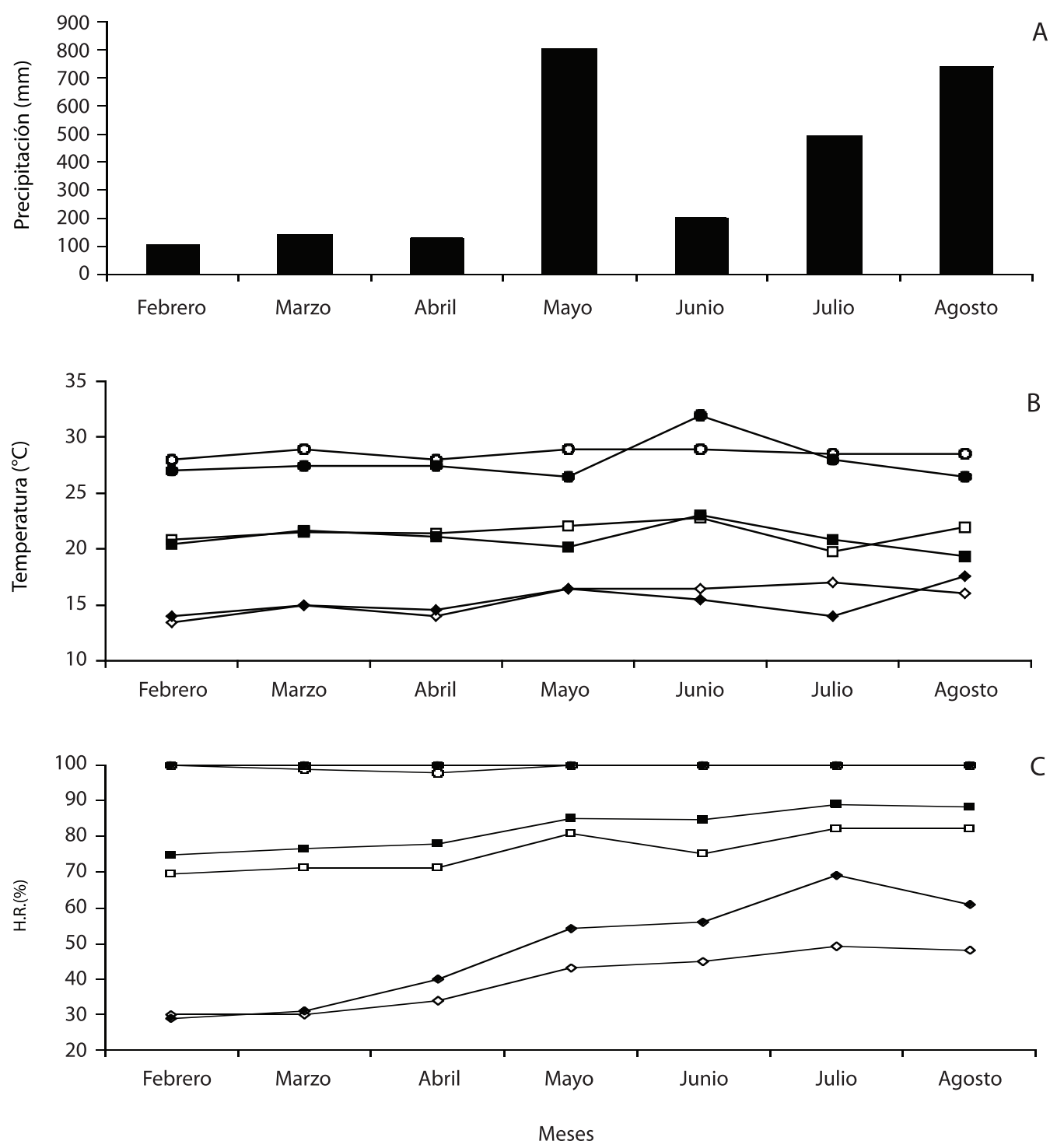

\begin{tabular}{|llll|}
\hline \multirow{3}{*}{ SOL } & mínimo $\diamond$ & & mínimo \\
& máximo $\bigcirc$ & SOMBRA & máximo \\
promedio $\square$ & & promedio \\
\hline
\end{tabular}

Fig. 2. Valores mensuales de precipitación (A.), temperatura (B.) y humedad relativa (C.) en cafetales con las variedades Caturra y Catimor, Santa Rosa de Turrialba, Costa Rica, 2002.

de cartón absorbente blanco, de $28 \mathrm{~cm}^{2}$, previamente sumergidos en una solución de aceite con atún (Barbera et al. 2004) y posteriormente mezclados con miel. Se tomaron 96 muestras en total, por cada muestreo, subdivididas en 48 estaciones de muestreo y en dos sitios en cada estación (suelo y estrato intermedio del arbusto de café).

Se realizaron observaciones mesoclimáticas solamente en la parcela I, debido a limitaciones 


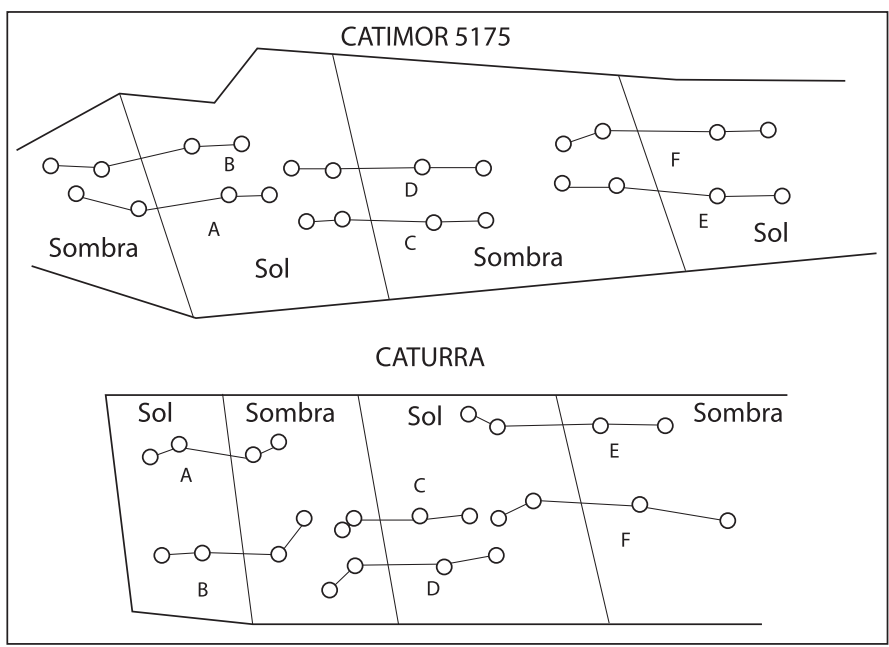

Fig. 1. Transectos con los cuatro puntos de muestreo a través del gradiente de luz-sombra en el experimento de distribución espacio-temporal de hormigas, Lote "El Cañal", Santa Rosa de Turrialba, Costa Rica, 2002. correspondió al suelo y al arbusto de café (en el estrato intermedio). La variable de respuesta fue la abundancia de cada una de las especies de hormigas en relación con los diferentes niveles de luz. Hubo seis repeticiones (transectos) dentro de cada medición mensual, para cada variedad de café, y siete mediciones mensuales durante el estudio (febreroagosto). El análisis estadístico realizado fue un análisis de varianza (ANOVA) (SAS Institute 1988). logísticas. Estas incluyeron mediciones diarias, continuas, de temperatura y humedad relativa del aire, mediante un higrotermógrafo (Maxant), en una de las subparcelas de sombra y en otra de sol. La precipitación se midió con un pluviómetro, en una de las subparcelas de sol.

En cuanto al análisis estadístico, se utilizó un diseño experimental de parcela dividida, dentro de un bloque completo al azar (SAS Institute 1988). La parcela principal fue la estación dentro de cada transecto, y la parcela pequeña fue el nivel de muestreo (sitio), que

\section{RESULTADOS}

En total se capturaron 28 especies, de las cuales las más abundantes fueron las siguientes seis: S. geminata, P. radoszkowskii, C. curvispinosa, P. cocciphaga, S. picea y Wasmania auropunctata (Cuadro 1). S. geminata alcanzó el $79 \%$ del total de individuos capturados en ambas parcelas; fue más abundante en la parcela con la var. Catimor, donde alcanzó el $90 \%$, mientras que en la otra (var. Caturra) alcanzó el $48 \%$. Por su parte, P. radoszkowskii alcanzó el $16 \%$ del total de individuos y fue más

CUADRO 1

Número de individuos capturados en las trampas, por especie de hormigas, en los muestreos realizados durante el periodo de estudio febrero-agosto, en dos variedades de café, Santa Rosa de Turrialba, Costa Rica, 2002

\begin{tabular}{|c|c|c|c|c|c|c|}
\hline \multirow[t]{2}{*}{ Especie } & \multicolumn{2}{|c|}{ var. Catimor } & \multicolumn{2}{|c|}{ var. Caturra } & \multirow[t]{2}{*}{ Total } & \multirow[t]{2}{*}{$\%$} \\
\hline & Sol & Sombra & Sol & Sombra & & \\
\hline S. geminata & 10780 & 3149 & 2265 & 375 & 16569 & 78.9 \\
\hline P. radoszkowskii & 471 & 432 & 831 & 1660 & 3394 & \\
\hline C. curvispinosa & 274 & 1 & 25 & 0 & 300 & 1.4 \\
\hline P. cocciphaga & 60 & 150 & 32 & 20 & 262 & 1.24 \\
\hline S. picea & 12 & 27 & 48 & 108 & 195 & 0.93 \\
\hline W. auropunctata & 13 & 38 & 71 & 12 & 134 & 0.3 \\
\hline Otras morfoespecies (22) & \multicolumn{2}{|c|}{105} & \multicolumn{2}{|c|}{38} & 143 & 0.8 \\
\hline Total & \multicolumn{2}{|c|}{15512} & \multicolumn{2}{|c|}{5485} & 20997 & \\
\hline
\end{tabular}


abundante en la parcela con la var. Caturra (45 $\%$ ); en la otra apenas alcanzó el $6 \%$.

Hubo tres especies (C. curvispinosa, P. cocciphaga y $S$. picea) que mostraron bajos niveles poblacionales, de apenas 1-1.5\%. C. curvispinosa alcanzó el $1.5 \%$ del total de individuos capturados y fue más abundante en la parcela con la var. Catimor $(1.7 \%)$ que en la otra $(0.5$ \%). P. cocciphaga alcanzó el $1.3 \%$ del total de individuos, pero fue más abundante en la parcela con la var. Caturra $(1.4 \%)$ que en la otra $(1 \%)$. S. picea alcanzó el $1 \%$ del total de individuos y fue más abundante en la parcela con la var. Catimor (3\%) que en la otra $(0.3 \%)$.

Asimismo, hubo 23 especies (22 de ellas no identificadas, y clasificadas como morfoespecies), encabezadas por $W$. auropunctata, que aparecieron en cantidades mínimas. Esta última alcanzó el $0.6 \%$ del total de individuos y fue más abundante en la parcela con la var. Catimor $(1.5 \%)$ que en la otra $(0.3 \%)$. Las 22 especies restantes alcanzaron apenas el $0.7 \%$ del total de individuos.

En general, las poblaciones de las tres especies selectas variaron marcadamente durante el período de estudio. Así, S. geminata mostró inicialmente en las dos variedades niveles altos de población, y tendió a decrecer conforme avanzó el estudio (Fig. 3A, B), aunque tuvo un leve repunte en agosto. Por su parte, $P$. radoszowskii tuvo una tendencia similar a la anterior especie en la var. Catimor, pero en la var. Caturra fue diferente, pues decreció de febrero a mayo, y aumentó hacia el final del estudio (junio-agosto) (Fig. 3C, D). Finalmente, C. curvispinosa tuvo una tendencia muy diferente a ellas, pues inicialmente sus poblaciones fueron muy bajas, crecieron hasta mayo, y luego tendieron a disminuir (Fig. 3E, F).

El efecto del gradiente de sol-sombra sobre la distribución y abundancia de las especies de hormigas, no se percibió de manera equivalente para las tres especies. En el caso de $S$. geminata, sus poblaciones fueron mucho más altas en la parcela con la var. Catimor que en la otra (Fig. 3A, B). En dicha parcela no mostró un patrón de abundancia congruente en ninguno de los gradientes, aunque sí tendió a perder importancia conforme avanzó el estudio (Fig. 3A). Inicialmente (febrero-marzo) fue más abundante en la subparcela SOP, y después en la SOL por los siguientes tres meses (abriljunio). En los dos meses finales lo fue en SOM, aunque en esta parcela había sido abundante en marzo. Por su parte, en la parcela con la var. Caturra fue mucho más abundante en SOL desde el principio (febrero-mayo) (Fig. 3B); hacia el final del estudio (agosto) solamente apareció en SOP.

Al analizar el número de individuos de $S$. geminata como un consolidado para todos los meses, en la subparcela con la var. Catimor hubo diferencias para el gradiente de luz, el sitio y el transecto, por separado $(p<0.05)$, pero no para sus interacciones. Para el primero, hubo preferencia por el nivel SOP, el cual no difirió de los niveles SOL y SMP $(p>0.05)$, pero sí de SOM $(p<0.05)$. Asimismo, para el sitio hubo diferencias $(\mathrm{p}<0.05)$, con preferencia por el suelo. Finalmente, también las hubo entre transectos $(p<0.05)$, con preferencia por el A en relación con $\mathrm{C}$ y D $(\mathrm{p}<0.05)$, pero igual a los transectos B, E y F ( $>>0.05)$.

Para la subparcela con la var. Caturra hubo diferencias para el gradiente de sombra y el transecto $(\mathrm{p}<0.05)$, pero no para la interacción. Para el primero hubo preferencia por el nivel SOL, el cual no difirió de los niveles SOP y SOM ( $>0.05)$, pero sí de SMP $(p<0.05)$. Además hubo diferencias para el transecto $(\mathrm{p}<0.05)$, con preferencia por el A en relación con B, D y F $(p<0.05)$, pero igual $(p>0.05)$ a los transectos $\mathrm{C}$ y $\mathrm{E}$.

Por su parte, P. radoszkowskii mostró valores poblacionales similares en ambas parcelas (Fig. 3C, D). Al considerar el efecto de las diferentes variables estudiadas, en la parcela con la var. Catimor las poblaciones de P. radoszkowskii no mostraron diferencias para ningún factor individual ni para sus interacciones ( $>0.05$ ). En cambio, en la otra parcela (var. Caturra) sí las hubo. Así, las poblaciones no mostraron diferencias entre gradientes (variable sombra) en relación con la fecha, excepto en julio y agosto, cuando hubo preferencia por el nivel SMP $(\mathrm{p}<0.05)$. Asimismo, hubo 

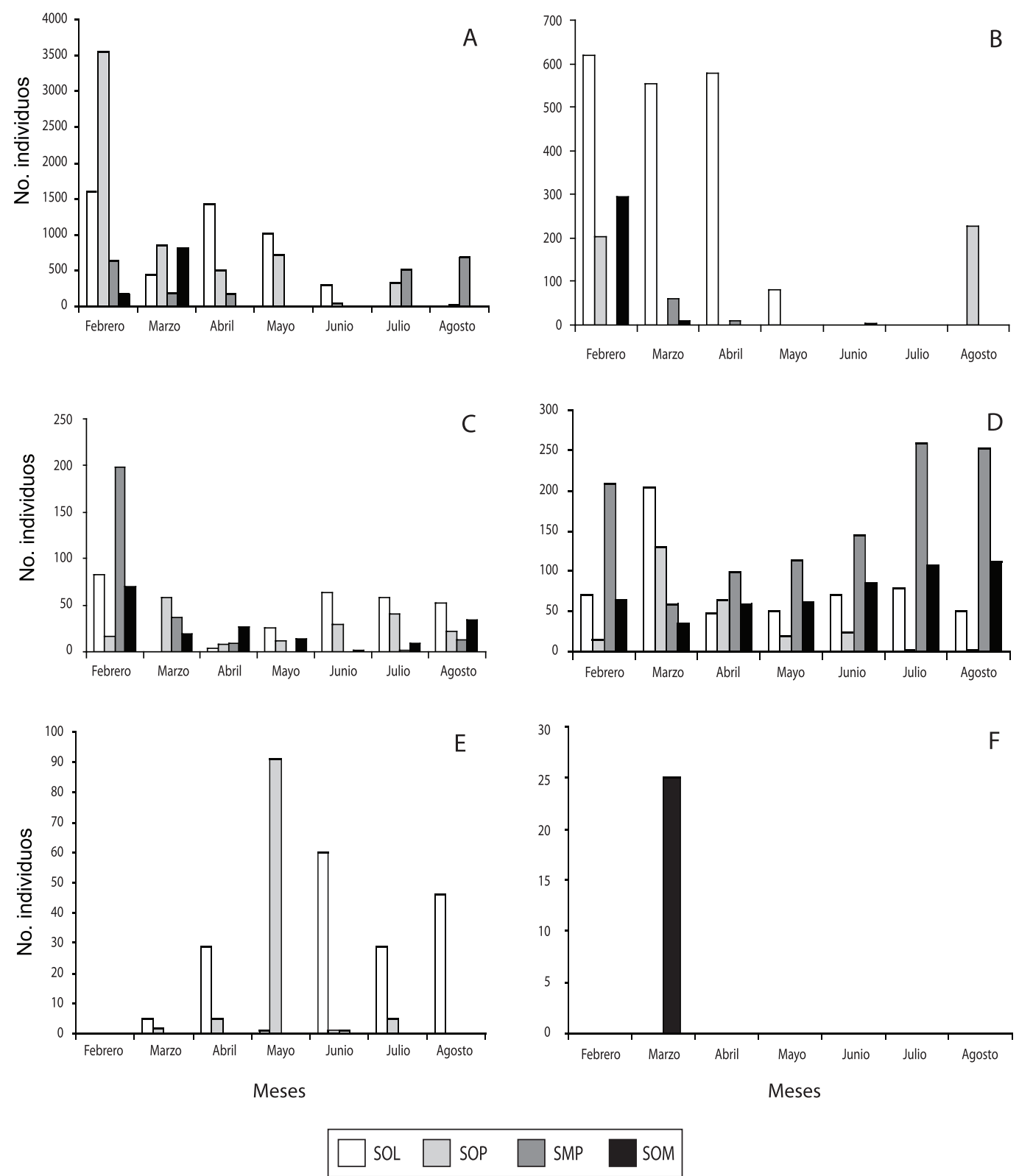

Fig. 3. Número de individuos de S. geminata (A, B), P. radoszkowskii (C, D) y C. curvispinosa (E, F) capturados en un gradiente de sol-sombra, en cafetales con las variedades Catimor (columna izquierda) y Caturra (columna derecha), Santa Rosa de Turrialba, Costa Rica, 2002.

varias interacciones significativas. En un caso fue para la interacción grado de luz-sitio, en abril $(\mathrm{p}<0.05)$, con preferencia por el suelo para los niveles SMP y SOM. En el otro caso, en junio y agosto las hubo para la interacción transecto-sombra $(\mathrm{p}<0.05)$, con los mayores valores para SMP en el transecto E.

Si se analiza el número de individuos de $P$. radoszkowskii como un consolidado para todos los meses, en la subparcela con la var. Catimor 
no hubo diferencias para ningún factor por separado ni para sus interacciones $(\mathrm{p}<0.05)$, pero para la var. Caturra las hubo para la interacción transecto-luz $(\mathrm{p}<0.05)$, con el mayor valor para el nivel SMP en el transecto $\mathrm{E}$ (Cuadro 2).

Finalmente, a pesar de sus bajos números, C. curvispinosa fue más abundante en la parcela con la var. Catimor, mientras que en la var. Caturra se capturó solamente una vez (Fig. 3E, F). En la primera apareció únicamente en las subparcelas SOL y SOP (Fig. 3E) y en la segunda en la subparcela SOL (Fig. 3F). En cuanto al sitio, en la primera parcela siempre prefirió el tronco (Fig. 4E), al igual que sucedió en la segunda, la única vez que fue detectada (Fig. 4F).

Al valorar el efecto de las diferentes variables estudiadas, en ninguna de las dos parcelas las poblaciones de C. curvispinosa mostraron diferencias para ningún factor individual ni para sus interacciones $(\mathrm{p}>0.05)$. No obstante, al considerar el número de individuos como un consolidado para todos los meses, en la subparcela con la var. Caturra no hubo diferencias para ningún factor por separado ni para sus interacciones $(p<0.05)$, pero sí las hubo para la var. Catimor, para el factor sitio $(\mathrm{p}<0.05)$, a favor del arbusto (Cuadro 2).

\section{DISCUSIÓN}

En cuanto a la composición de especies de la comunidad de hormigas estudiada, se capturaron 28 especies, cifra superior a la obtenida por Barbera et al. (2004), quienes hallaron 19 especies, en cafetales de la misma zona, aunque su estudio se efectuó a menor altitud (590 m). Asimismo, hubo coincidencia en que $S$. geminata fue la especie dominante, con el $79 \%$ del total de individuos, frente al $89 \%$ en el trabajo de dichos autores. Esta especie fue seguida por $P$. radoszkowskii, con el $16 \%$ y el $7 \%$, respectivamente.

De las otras cuatro especies identificadas aquí, también hubo coincidencias, pues tres de ellas (P. cocciphaga, S. picea y W. auropunctata) estuvieron entre las seis especies más abundantes en el estudio de Barbera et al. (2004). Las únicas diferencias evidentes fueron la presencia de Tapinoma paratrachina, que ocupó el cuarto lugar en dicho estudio y aquí ocupó el noveno lugar, y la bajísima presencia de $C$. curvispinosa, que en aquél ocupó el lugar 19 y aquí se ubicó en el tercer lugar. Esto último pudo obedecer a que se utilizó un cebo diferente al de dichos autores, compuesto por atún-aceite-miel (Romero y Jaffé 1989), para que fuera más atractivo para dicha especie.

CUADRO 2

Relaciones de significancia estadística entre los recuentos totales (durante el período de estudio) del número de individuos de tres especies de hormigas y cuatro grados de luz, dos sitios de muestreo y sus interacciones, según la variedad de café, Santa Rosa de Turrialba, Costa Rica, 2002

Grado luz Sitio Transecto Luz-Sitio $\quad$ Transecto-Luz

var. Catimor

S. geminata

P. radoszkowskii

* *

C. curvispinosa

NS

NS

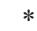

NS

NS

var. Caturra

NS

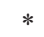

NS

NS

NS

S. geminata

P. radoszkowskii

* NS

NS

NS

NS

C. curvispinosa

$$
\text { NS }
$$

NS

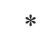

NS

NS

NS

NS

*

NS

NS

NS

Grado de luz $=$ transecto en sol $(\mathrm{Sol})$, transecto en sombra (Sombra).

Nivel: No significativo (NS); significativo $(*)(\mathrm{p}<, 05)$; y altamente significativo $\left(^{* *}\right)(\mathrm{p}<, 01)$. 

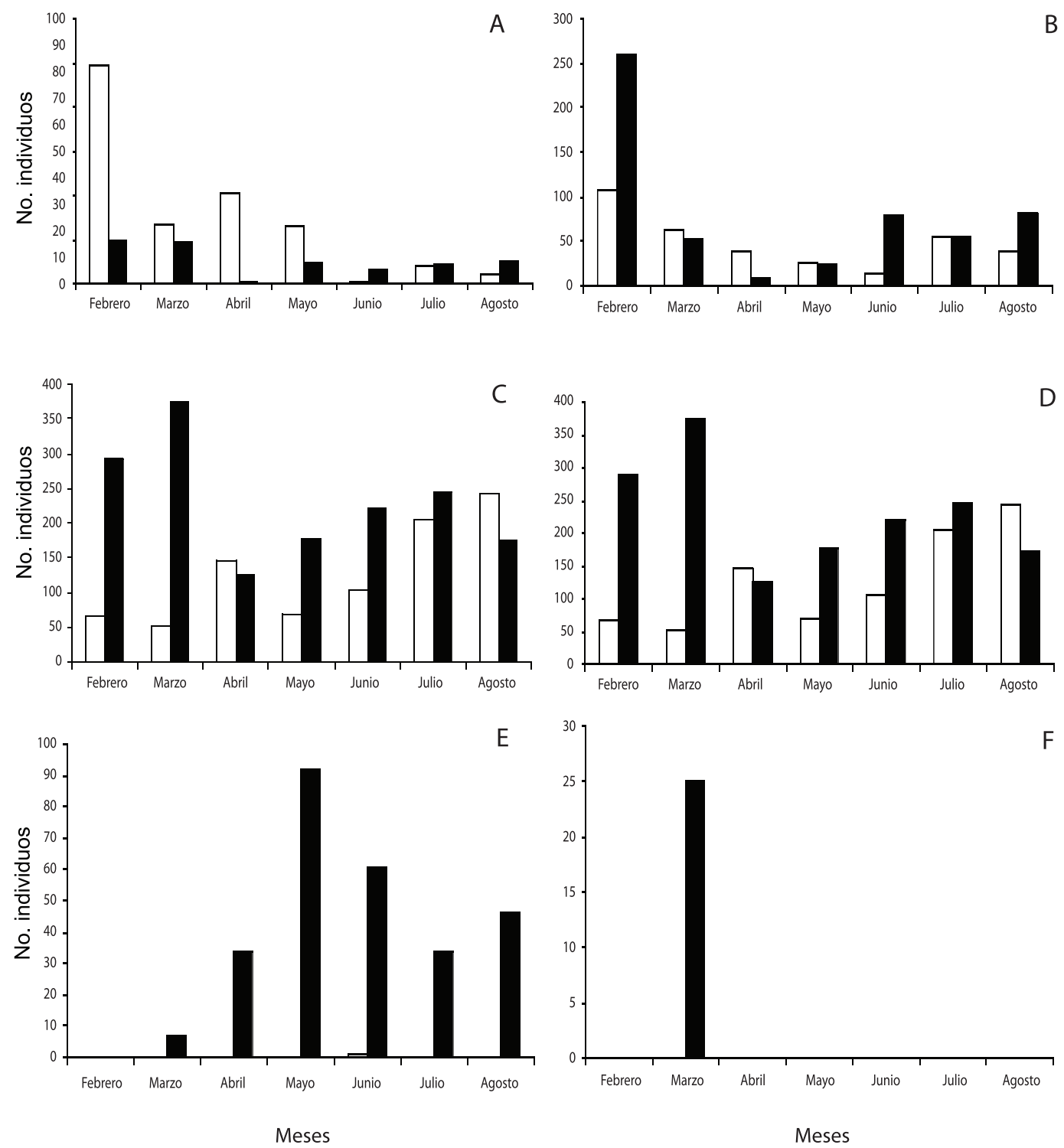

\section{SUELO}

ARBUSTO

Fig. 4. Número de individuos de S. geminata (A, B), P. radoszkowskii (C, D), C. curvispinosa (E, F) capturados en dos sitios, en cafetales con las variedades Catimor (columna izquierda) y Caturra (columna derecha), Santa Rosa de Turrialba, Costa Rica, 2002.

La abundancia de $S$. geminata y $P$. radoszkowskii fue mucho menor que en el estudio de dichos autores, lo cual podría deberse a varios factores. El primero fue la cantidad de muestras, pues aquí la periodicidad de muestreo fue mensual y no quincenal, y además se tomó un menor número de muestras por fecha; aquí fueron apenas 96 para las dos variedades, mientras que ella tomó 270 muestras en cada fecha de muestreo.

Otro factor, menos evidente, fue el diseño utilizado, ya que se muestrearon transectos 
permanentes. Esto podría generar sesgos, ya que al trasladar una especie sus nidos hacia otro sitio, tal colonia se tomaría por perdida cuando, en realidad, hubo tan solo una mudanza de sitio. Esto también fue informado por Romero y Jaffé (1989), quienes al muestrear repetidamente con trampas de foso en un mismo sitio, notaron que el número de nuevas especies recolectadas disminuyó con el tiempo y, además, el número de individuos de las especies recolectadas decreció. En cambio, Barbera et al. (2004) realizó muestreos al azar, lo cual aumentaría la probabilidad de hallar más nidos en cierta área y así aumentar la captura en las trampas. En todo caso, para conocer mejor las causas de los cambios en las poblaciones, además de las capturas en trampas, se podría haber muestreado la abundancia de nidos de estas especies, así como la cantidad de hojarasca presente en el suelo.

Desde una perspectiva aplicada, las dos especies de hormigas dominantes ( $S$. geminata y $P$. radoszkowskii) tienen la capacidad de depredar tanto a la broca del café como al barrenador de las meliáceas; mientras que $C$. curvispinosa lo puede hacer sobre la broca (Varón et al. 2004, 2005). Por tanto, dado su potencial en el control biológico de estas plagas, ameritaría favorecer su conservación e incremento, mediante el manejo de su hábitat, el cual pareciera ser afectado por la presencia de árboles de sombra en los cafetales.

En este caso, en cuanto a la respuesta de las especies de hormigas a diferentes grados de sombra, así como a las condiciones microclimáticas resultantes de éste, las parcelas de café de ambas variedades tuvieron diferentes características, aunque estaban ubicadas en una misma finca. En la parcela con la var. Caturra había mayor densidad de plantas, con distancias de siembra de $1.7 \mathrm{~m}$ entre hileras y $1.2 \mathrm{~m}$ entre plantas, mientras que en la de la var. Catimor eran de $2 \times 1.2 \mathrm{~m}$, lo cual creó condiciones evidentes de mayor autosombra para la primera. Debido a que se registraron datos climáticos solamente para la primera parcela, no fue posible cuantificar las diferencias en las condiciones climáticas entre ambas.
En cuanto a su población total, es claro que la mayor autosombra del café en la var. Caturra favoreció más a $P$. radoszkowskii, en contraste con la menor autosombra de la var. Catimor, que favoreció más $S$. geminata y a $C$. curvispinosa. De hecho, $P$. radoszkowskii en dicha variedad tuvo una población total casi tres veces mayor que en la parcela con la var. Catimor. En esta última, en cambio, las poblaciones de $S$. geminata y $C$. curvispinosa fueron unas siete y once veces mayores que en la otra, respectivamente.

Es interesante resaltar que $P$. radoszkowskii fue la especie dominante en condiciones de sombra para la var. Caturra, quintuplicando la población de $S$. geminata en dicha condición, al final del estudio. Este resultado es congruente con los de otros autores para $S$. geminata y $P$. radoszkowskii en la zona de Turrialba (Benítez y Perfecto 1989, Barbera et al. 2004), así como para otras zonas de Costa Rica (Perfecto y Vandermeer 1994, 1996, Perfecto y Snelling 1995). También se percibió un efecto estacional en la var. Caturra, observándose que cuando se presentó la menor precipitación (febreroabril) $S$. geminata fue dominante sobre $P$. radoszkowskii, en contraste con el período de mayor precipitación (mayo-agosto), cuando ésta alcanzó mayores poblaciones.

$S$. geminata suele establecer sus colonias o nidos en el suelo, pero al parecer no es muy exigente con respecto a los sitios de anidación (Perfecto y Vandermeer 1994), lo cual coincide con lo detectado en este estudio. Por observaciones realizadas en el área, sus nidos fueron fabricados con fragmentos de suelo principalmente, aunque además utilizó hojarasca y troncos muertos, al parecer aprovechando los recursos disponibles en la plantación.

Asimismo, S. geminata es más común en áreas abiertas, y más abundante en monocultivos que en plantaciones algo o muy diversificadas. Además, sus nidos son muy grandes, y típicamente incluyen una extensa área del subsuelo y de alimentación (Perfecto y Vandermeer 1996). En general, esto se reflejó en el presente estudio, detectándose en cada variedad las mayores poblaciones en las parcelas de sol. No obstante, 
quizás debido a la profusa cobertura de hojarasca, no hubo especies de gramíneas en las áreas de sol, en cuyas raíces es común hallar áfidos que aportan carbohidratos para las hormigas (Perfecto y Vandermeer 1994); por ello es que ellas defienden dichas plantas, normalmente con éxito (Perfecto y Vandermeer 1996).

Un hábito característico de esta especie fue su permanente cambio de sitios de anidación. Esto fue observado tanto en la parcela de estudio, como en Heredia, en el Valle Central del país (Varón, obs. pers.), donde frecuentemente aparecieron muchos nidos vacíos. Este comportamiento contrastó con el de las otras dos especies, que tuvieron más permanencia en sus nidos durante el estudio. En este sentido, Perfecto y Vandermeer (1996) observaron un movimiento de nidos de $S$. geminata dentro de las $24 \mathrm{~h}$ después de imponer sombra artificialmente en su área de estudio.

Por su parte, $P$. radoszkowskii usualmente ocupa una importante área en las plantaciones de café $y$, además de ser una eficiente reclutadora, tiene mayor capacidad para localizar recursos que $S$. geminata. El reclutamiento es un mecanismo de comunicación que permite atraer a miembros de la misma colonia a sitios donde su trabajo es necesario (Wilson 1971).

Asimismo, en contraste con las grandes y abundantes colonias de $S$. geminata, las de $P$. radoszkowskii son mucho más pequeñas. Esta se alimenta en varios sitios, encontrándose en cebos colocados tanto en el suelo como en los arbustos, pero anida en el suelo (Perfecto y Vandermeer 1994). No presenta una respuesta congruente en su preferencia por hábitats sombreados o soleados (Perfecto y Vandermeer 1996), como se documentó en este estudio, pues fue más abundante al sol en la var. Catimor, y a la sombra en la var. Caturra. No obstante, Benítez y Perfecto (1989) informaron que a mayor intensidad de luz la actividad de esta especie era menor.

Aunque el lote El Cañal tenía parcelas alternas de sombra y de sol, debido al gran tamaño de los árboles, que superaban los $20 \mathrm{~m}$, su hojarasca también cubría gran parte de las parcelas de sol. Por tanto, esto quizás aportó sustrato para que $P$. radoszkowskii construyera sus nidos. Sin embargo, en un experimento de depredación sobre H. hampei efectuado en Heredia (Varón, obs. pers.) los nidos estaban construidos con fragmentos de suelo, quizás debido a la escasez de hojarasca en esa plantación.

Estos hallazgos coinciden con los argumentos de Perfecto y Vandermeer (1994) con respecto a que los sitios de anidación no parecen influenciar la distribución de $S$. geminata ni de $P$. radoszkowskii. En este estudio los nidos de ambas especies fueron construidos con los materiales disponibles y, aunque había hojarasca tanto en áreas de sombra como de sol, los patrones de abundancia de ambas especies se mantuvieron, siendo $S$. geminata más abundante en áreas soleadas y $P$. radoszkowskii alcanzando poblaciones similares en las dos condiciones.

Finalmente, C. curvispinosa apareció casi exclusivamente en la parte aérea de los arbustos de café y en áreas soleadas. Sus nidos estaban principalmente en grietas del tronco asociadas con los cortes de las podas del arbusto, dentro de las cuales hacen pequeñas cámaras en el tallo. Además, no apareció en los árboles de poró, en contraste con las otras dos especies. Sin embargo, también se encontraron nidos construidos sobre el suelo, dentro de ramas secas huecas o de troncos cortados caídos (Varón, obs. pers.). Este comportamiento flexible de anidación coincide con el de otras especies congéneres (Majer 1972, Byrne 1994, Soria et al. 1994).

Es importante destacar que en el presente estudio se realizó un análisis del sitio de la parcela en el cual son activas las especies de hormigas estudiadas, con el interés práctico de conocer cuál es el área en que podría expresar su actividad depredadora. Esto es importante porque $H$. hampei aparece en los frutos frescos cuando la planta está en plena producción, y posteriormente en los granos viejos que caen al suelo. En cada caso, podría ser importante conocer cuál especie utilizar, según la temporada del cultivo.

Por ejemplo, C. curvispinosa fue casi exclusivamente arbórea, aunque puede aparecer 
sobre el suelo, dentro de ramas secas huecas. En cambio, aunque $S$. geminata apareció tanto sobre el suelo como en el arbusto, mostró una leve preferencia por el suelo, sobre todo en la var. Catimor. Por su parte, aunque P. radoszkowskii apareció en ambos sitios, mostró una leve preferencia por el arbusto, lo cual fue más acentuado en la var. Caturra.

Perfecto y Snelling (1995) indican que, en la mayoría de estudios sobre ecología de comunidades, éstas son definidas en función del método de muestreo empleado. Por tanto, señalan que es muy probable que las hormigas encontradas en la superficie de los arbustos de café incluyan tanto generalistas que buscan alimento en el suelo, como algunas especies arbóreas, pues las hormigas terrestres suben por las ramas del café, comúnmente. En este sentido, tanto $S$. geminata como $P$. radoszkowskii anidan en el suelo y son consideradas hormigas terrestres, pero aparecen en los arbustos de café y en los árboles de poró. Por su parte, C. curvispinosa puede considerarse como arbórea, debido a sus hábitos de anidación en los arbustos de café, aunque en una ocasión se le halló en un muestreo realizado en el suelo.

Asimismo, el efecto de las condiciones microclimáticas presentes en el gradiente de sol-sombra sobre la abundancia y distribución de las especies de hormigas no se percibió de manera equivalente para las tres especies. Una evidencia clara del efecto del grado de sombra sobre las poblaciones de hormigas fue que éstas fueron mucho mayores para $S$. geminata y $C$. curvispinosa en la parcela menos densa (var. Catimor) y para $P$. radoszkowskii en la más densa (var. Caturra).

A pesar de que en El Cañal había un sistema maduro, cercano a 10 años de edad y con árboles altos de poró, $S$. geminata y $P$. radoszkowskii fueron las especies dominantes tanto en las áreas de sombra como de sol. En efecto, aunque las poblaciones de S. geminata disminuyeron en las áreas sombreadas, su dominancia en esta condición se mantuvo en la var. Catimor, así como en las áreas soleadas en las dos variedades. Por su parte, $P$. radoszkowskii fue la segunda especie en importancia en las dos condiciones, excepto en la var. Caturra en condiciones de sombra, donde fue la especie dominante.

En contraposición con lo planteado por Benítez y Perfecto (1989), la combinación de estas dos especies no parece exclusiva de etapas tempranas de sucesión en las comunidades de hormigas, sino que más bien dicha dominancia se mantiene mientras haya arbustos de café junto con árboles de poró.

Curiosamente, Barbera et al. (2004) encontraron que en cafetales recién establecidos, con menos de un año de edad, así como en un cafetal comercial con cinco años, S. geminata fue dominante de manera masiva, pero esta situación se invirtió a favor de $P$. radoszkowskii en un cafetal de siete años de edad; asimismo, $P$. cocciphaga tuvo una población muy cercana a la de $S$. geminata.

Ellos lo explicaron en el sentido de que $S$. geminata es más común en áreas abiertas, así como más abundante en monocultivos que en plantaciones algo o muy diversificadas, posiblemente porque en estas áreas, donde las gramíneas colonizan rápidamente, es común que algunas especies de áfidos presentes en sus raíces sean un recurso importante para $S$. geminata (Perfecto y Vandermeer 1994). Asimismo, porque las condiciones de alta humedad y hojarasca aportada por los árboles de sombra no le favorecen en sus labores de anidación.

Para $S$. geminata hubo mayores poblaciones en las áreas de sol, presentando al final del estudio mayor abundancia para dichas áreas en ambas variedades. $P$. radoszkowskii no mostró un patrón congruente de preferencia por cierto nivel de sombra; en la var. Caturra tuvo mayores poblaciones en las áreas de sombra, pero en la var. Catimor fue más abundante en áreas de sol. Estos resultados coinciden con Perfecto y Vandermeer (1996), quienes indicaron que las poblaciones de $S$. geminata decrecieron por efecto de la sombra y P. radoszkowskii no mostró una respuesta congruente al cambio en los niveles de sombra. Por su parte, C. curvispinosa mostró mayor abundancia en las parcelas de sol en ambas variedades.

A pesar de que hubo mayores poblaciones de C. curvispinosa y $S$. geminata en las 
parcelas de sol (SOL y SOP) comparadas con las de sombra (SMP y SOM), la diferencia no fue significativa en términos estadísticos. Este hecho se puede explicar porque, dadas las diferencias entre los transectos, en general ambas especies aparecieron en pocos de los transectos establecidos, y éstos respondieron por toda la población encontrada en los muestreos. Ello se reflejó en las leves diferencias estadísticas detectadas.

En efecto, al constatar que en varios meses hubo significancia para la interacción transectogrado de luz y al observar los datos primarios, se puede concluir que el efecto del transecto tuvo un gran peso en los resultados. Esto fue más claro en el caso de $S$. geminata y $C$. curvispinosa, en los que gran parte del número de individuos capturados en los muestreos fueron capturados en uno o dos de los transectos.

Otro aspecto importante en este estudio fue que el número de individuos no fue un buen indicador de la frecuencia de captura de la especie. Por ejemplo, aunque $S$. geminata siempre fue más abundante que $P$. radoszkowskii, y siempre se capturaron cientos de individuos, apareció menos veces (menor frecuencia) que ésta.

Es interesante que el número de nidos claramente fue mayor para $P$. radoszkowskii, según observaciones ocasionales. Este aspecto guarda similitud con lo observado en Venezuela (Romero y Jaffé 1989), donde Labidus coecus fue recolectada solamente unas pocas veces, aunque cada vez miles de individuos llenaban las trampas; asimismo, las obreras de Atta spp., fueron atrapadas en muy bajos números, pero una única obrera atrapada indicaba la presencia de grandes nidos de esta especie. Por tanto, a criterio de estos autores, el número de individuos y de nidos no fueron buenos indicadores de abundancia, mientras que la frecuencia de captura fue un indicador más confiable.

De hecho, $P$. radoszkowskii fue la especie que apareció con mayor frecuencia en los muestreos realizados, en los diferentes transectos y niveles de sombra. En la var. Catimor fue encontrada 75 veces, mientras que $S$. geminata lo fue 52 veces y $C$. curvispinosa 17 veces; en la var. Caturra aparecieron 121, 23 y una vez, respectivamente. Es decir, P. radoszkowskii estaba distribuida por toda el área del estudio, aunque debido al tamaño de sus colonias sus números finales fueron inferiores a los de $S$. geminata.

En general, las especies estudiadas presentaron tendencias de distribución similares a las informadas en la literatura (Benítez y Perfecto 1989, Perfecto y Vandermeer 1994), pues $S$. geminata fue más abundante en condiciones soleadas, mientras que $P$. radoszkowskii fue igualmente abundante en condiciones de sombra y de sol. Con respecto a C. curvispinosa, para la cual no había referencias al respecto, mostró mayor abundancia por condiciones soleadas. Dentro de los factores abióticos posiblemente responsables por estas preferencias, la humedad podría haber jugado un papel importante, debido a que entre las condiciones estudiadas fue la que más difirió entre los microhabitats de sombra y sol.

\section{AGRADECIMIENTOS}

A Sérge Savary y Nadiejda Barbera (CATIE), así como a Sanford Eigenbrode (University of Idaho), por sus consejos y aportes en el planeamiento y desarrollo de la investigación. A Arturo Ramírez, Asdrúbal Chavarría, Arturo Gamboa, Claudio Arroyo, Guido Sanabria, Rodrigo Granados, por su apoyo en la recolección de datos en el campo, así como a Daniel Coto y Sergio Velásquez (CATIE), por su apoyo logístico. A Gilberto Páez y Gustavo López, por su colaboración en aspectos estadísticos.

\section{RESUMEN}

En los sistemas agroforestales de café (Coffea arabica L.) de Mesoamérica es frecuente la presencia de árboles de sombra. Estos sistemas pueden albergar una rica entomofauna, incluyendo hormigas, la cuales podrían depredar a plagas claves en dichos sistemas. Sin embargo, se desconoce el papel de la sombra sobre la distribución y abundancia de dichas hormigas, lo cual podría sugerir 
pautas para manipular ciertas condiciones de su hábitat y lograr su conservación e incremento. Por tanto, se estudió el efecto de la sombra sobre la distribución espacial y temporal de tres especies de hormigas (Solenopsis geminata, Pheidole radoszkowskii y Crematogaster curvispinosa) que pueden depredar a la broca del café, Hypothenemus hampei (Coleoptera: Scolytidae) y al barrenador de las meliáceas, Hypsipyla grandella. Para ello se evaluó su abundancia a través de un gradiente de sol-sombra en un cafetal con cuatro parcelas alternas (desde pleno sol hasta sombra total), en Turrialba, Costa Rica. En la comunidad estudiada se recolectaron 28 especies de hormigas, de las cuales $S$. geminata fue la dominante $(79 \%$ del total de individuos), seguida por $P$. radoszkowskii (16 \%). S. geminata y C. curvispinosa prefirieron las áreas soleadas, mientras que $P$. radoszkowskii no mostró una preferencia definida. Asimismo, en cuanto a su ubicación, S. geminata predominó en el suelo, mientras que $P$. radoszkowskii y $C$. curvispinosa lo hicieron en los arbustos de café.

Palabras clave: Café, sombra, Solenopsis geminata, Pheidole radoszkowskii, Crematogaster curvispinosa, Costa Rica, sistemas agroforestales.

\section{REFERENCIAS}

Barbera, N., L. Hilje, P. Hanson, J.T. Longino, M. Carballo \& E. de Melo. 2004. Diversidad de especies de hormigas en un gradiente de cafetales orgánicos y convencionales. Man. Integr. Plag. Agroecol. 72: 60-71.

Beer, J., R. Muschler, D. Kass \& E. Somarriba. 1998. Shade management in coffee and cacao plantations. Agrofor. Syst. 38: 139-164.

Benítez, J. \& I. Perfecto. 1989. Efecto de diferentes tipos de manejo de café sobre las comunidades de hormigas. Agroecol. Neotrop. 1: 11-15.

Byrne, M.M. 1994. Ecology of twig-dwelling ants in a wet lowland tropical forest. Biotropica 26: 61-72.

Hölldobler, B. \& E.O. Wilson. 1990. The ants. Harvard University, Boston, Massachussets, EEUU. 571 p.

Huang, H.T. \& P. Yang. 1987. The ancient cultured citrus ant: A tropical ant is used to control insect pests in Southern China. Bioscience 37: 665-671.

Longino, J.T. \& P.E. Hanson. 1995. The ants (Formicidae), p. 589-620. In P.E. Hanson \& I.D. Gauld (eds.). The
Hymenoptera of Costa Rica. Oxford and Nat. Hist. Mus., Londres Inglaterra.

Majer, J.D. 1972. The ant mosaic in Ghana cocoa farms. Bull. Entomol. Res. 62: 151-160.

Nestel, D., F. Dickschen \& M. Altieri. 1992. Diversity patterns of soil macro-Coleoptera in Mexican shade and unshaded coffee agroecosystem: an indication of habitat perturbation. Biodiv. Conserv. 2: 70-78.

Perfecto, I. \& A. Castiñeiras. 1998. Deployment of the predaceous ants and their conservation in agroecosystems, p. 269-289. In P. Barbosa (ed.). Conservation biological control. Academic, Washington DC, EEUU.

Perfecto, I. \& R. Snelling. 1995. Biodiversity and the transformation of a tropical agroecosystem: Ants in coffee plantations. Ecologic. Applic. 5: 1084-1097.

Perfecto, I. \& J. Vandermeer. 1994. Understanding biodiversity loss in agroecosystems. Reduction of ant diversity resulting from transformation of the coffee ecosystem in Costa Rica. Entomol. Trends Agril. Sci. 2: 7-13.

Perfecto, I. \& J. Vandermeer. 1996. Microclimatic changes and the indirect loss of ant diversity in a tropical agroecosystem. Oecologia. 108: 577-582.

Perfecto, I., J. Vandermeer, P. Hanson \& V. Cartín. 1997. Arthropod biodiversity loss and the transformation of a tropical agro-ecosystem. Biodiv. Conserv. 6: 935-945.

Rojas, L., C. Godoy, P. Hanson \& L. Hilje. 2001a. A survey of homopteran species (Auchenorrhyncha) from coffee shrubs and poró and laurel trees in shaded coffee plantations, in Turrialba, Costa Rica. Rev. Biol. Trop. 49: 1057-1065.

Rojas, L., C. Godoy, P. Hanson, C. Kleinn \& L. Hilje. 2001b. Hopper (Homoptera: Auchenorrhyncha) diversity in shaded coffee systems of Turrialba, Costa Rica. Agrofor. Syst. 53: 171-177.

Romero, H. \& K. Jaffé. 1989. A comparison of methods for sampling ants (Hymenoptera: Formicidae) in savannas. Biotropica 21: 348-352.

SAS Institute. 1988. SAS language guide for personal computers. SAS Institute, Cary, Carolina del Norte, EEUU. 558 p. 
Soria, F.J., M. Villagrán \& M.E. Ocete. 1994. Estudio del comportamiento alimentario de Crematogaster scutellaris Oliv. (Hym., Formicidae) en tres alcornocales del SW español. Bolet. Sani.Veget. Plag. 20: 637642 .

Varón, E.H., P. Hanson, O. Borbón, M. Carballo \& L. Hilje. 2004. Potencial de hormigas como depredadoras de la broca del café (Hypothenemus hampei) en Costa Rica. Man. Integr. Plag. 73: 42-50.
Varón, E.H., N. Barbera, P. Hanson, M. Carballo \& L. Hilje. 2005. Potencial de depredación de Hypsipyla grandella por hormigas, en cafetales de Costa Rica. Man. Integr. Plag. 74: 17-23.

Way, M.J. \& K.C. Khoo. 1992. Role of ants in pest management. Ann. Rev. Entom. 37: 479-503.

Wilson, E.O. 1971. The insect societies. Harvard University, Boston, , Massachussets, EEUU. 548 p. 